\title{
Pattern Recognition Receptors (PRRs)
}

\author{
Joycee Jogi*, Varsha Sharma, Anju Nayak, Vandana Gupta, \\ Poonam Shakya and Smita Bordoloi
}

\begin{abstract}
Department of Veterinary Microbiology, College of Veterinary Science \& A. H. N.D.V.S.U., Jabalpur (M.P.), India
\end{abstract}

*Corresponding author

\begin{abstract}
A B S T R A C T
Pattern recognition receptors (PRRs) play a very important role in the innate immune response by identifying the core structures called as pathogen associated molecular patterns (PAMPs) distinctive to each pathogen and these obligatory structures are also

Keywords

Pattern recognition receptors (PRRs),

Pathogen associated

molecular patterns

(PAMPs), Damage-

associated molecular

patterns (DAMPs)

Article Info

Accepted:

24 August 2018

Available Online:

10 September 2018 required for the survival of pathogens. PRRs play a vital role by recognizing PAMPs which are distinct to each pathogen, and are fundamental molecular structures required for the pathogens survival. PRRs are mainly expressed by macrophage and dendritic cells and determine assorted group of pathogens. They activate pro-inflammatory signaling pathways, stimulate phagocytic responses or bind to micro-organisms as secreted proteins. When our body senses that it is under attack the innate immune system gets activated. This is because our body recognizes alarm signals generated by two pathways. First is the exogenous alarm signals which are generated by invading microorganisms and are collectively known as PAMPs. Whereas the endogenous alarm signals are produced by dead and dying cells and are called as damage-associated molecular patterns (DAMPs) which are excreted or released by dead, dying and damaged cells. The PRRs recognize DAMPs and PAMPs and activate the innate immune system. Recent studies reveal that the innate immune system has a greater specificity and is highly developed in its ability to discriminate between self and foreign. These responses are not only essential for early pathogen recognition, but are also involved in the activation and shaping of adaptive immunity.
\end{abstract}

\section{Introduction}

Infectious agents such as bacteria and viruses multiply rapidly. If these microbes invade the body, they must be recognized and destroyed before they can overwhelm the defenses. The body must therefore employ fast-reacting mechanisms as its first line of defense against invaders. These mechanisms constitute the innate immune system. Innate immune mechanisms have arisen in different ways and at different times in response to different threats (Gardy et al., 2009). As a result, the innate immune system consists of many diverse subsystems. The most important of these innate subsystems is the process of inflammation. Inflammation triggers the migration of leukocytes, from the bloodstream to sites of invasion where they attack and destroy invaders. It is therefore a mechanism 
by which defensive cells and proteins are focused on sites of microbial invasion. Together, they destroy the invaders and then repair any subsequent tissue damage (Medzhitov, 2008).

\section{Activation of innate immune system}

Immunologists over the world has gradually shifted their focus from antigen-specific $\mathrm{T}$ and $B$ cells receptors to a set of germ line encoded receptors that play important roles in innate immunity. The innate immune system is activated when the body senses that it is under attack (Medzhitov, 2007). This involves recognizing alarm signals generated by two pathways. Alarm signals are generated either by invading microorganisms (exogenous signals) or by dead and dying cells (endogenous signals). Exogenous signals consist of molecules produced by microbial invaders. Collectively, these are called pathogen-associated molecular patterns (PAMPs).

Endogenous signals consist of molecules released from damaged, dead, or dying cells. These are collectively called damageassociated molecular patterns (DAMPs). Together, the DAMPs and PAMPs are recognized by PRRs on sentinel cells located throughout the body. Once recognized, they activate the innate immune system (Akira et al., 2007 and Bianchi, 2012).

Janeway (1989) suggested that PRRs play a very vital role in triggering adaptive immunity. The seminal work that identified the first PRR was provided by, (Lemaitre et al., 1996) when they demonstrated that mutant flies lacking a functional Toll protein were susceptible to fungal infections. This initiated a rush of studies to identify PRRs and to understand how innate immunity could initiate and shape adaptive immune responses. Since then, many surface and cytosolic PRRs have been identified in higher mammals and throughout evolution and to understand how signaling through these receptors allows for the initiation of adaptive immune responses.

PRRs expressed by innate immune cells are essential for detecting invading pathogens and initiating the innate and adaptive immune response.

\section{There are multiple families of PRRs including-}

Membrane-associated-

Toll-like receptors (TLRs)

C-type lectin receptors (CLRs),

Cytosolic-

NOD like receptors (NLRs)

RIG-I-like receptors (RLRs).

\section{Pattern recognition receptors}

PAMPs and DAMPs possess the ability to activate PRRs. The recognition of microbial molecules and damaged cells by PRR activates intracellular signal cascades which lead to the expression of pro-inflammatory cytokines, chemokines and antiviral molecules (Takeuchi and Akira, 2010). On the contrary, activation of some NLRs forms multiprotein inflammasome complexes that aids in the cleavage and activation of Caspase-1 (Schroder and Tschopp, 2010) which promotes the maturation and secretion of IL$1 \beta$ and IL-18, which is a part of proinflammatory immune response. A single pathogen can simultaneously activate multiple PRRs, crosstalk between different receptors may also play a role in enhancing or inhibiting the immune response (Kingeter and Lin, 2012). Therefore, a regular surveillance of PRR signaling is required in order to get rid of the virulent microorganisms and 
simultaneously check the excessive PRR activation, which can initiate the occurrence of inflammatory and autoimmune diseases (Mogensen, 2009). There are two major consequences of ligating PRRs on immune cells. The first is to signal danger to the cells and to initiate a cascade of responses that direct host defense responses. The second is to persuade the capability of selected cells to present antigen to $T$ cells. PRR stimulation initiates dendritic cell (DC) maturation to stabilize major histocompatibility complex (MHC) molecules on their surface and facilitates antigen presentation.

\section{Classification of PRRs}

PRRs are classified according to their ligand specificity, function, localization and/or evolutionary relationships. On the basis of function, PRRs may be divided into signaling and endocytic PRRs.

Signaling PRRs include the large families of membrane-bound Toll-like receptors and cytoplasmic NOD-like receptors.

Endocytic PRRs promote the attachment, engulfment and destruction of microorganisms by phagocytes, without relaying an intracellular signal.

\section{PRR types and localization}

\section{Membrane-bound PRRs}

\section{Receptor kinases}

PRRs, which are associated with intracellular kinases via adaptor proteins (Fig. 1).

\section{Toll-like receptors (TLR)}

TLRs are are a critical first line of defense which are located on cell surfaces, where they are responsible for recognizing extracellular invaders such as bacteria and fungi. Other TLRs are located within the cells, where they are responsible for detecting intracellular invaders such as viruses (Blasius and Beutler, 2010). They are expressed by many different cell types. Most importantly, they are expressed on sentinel cells such as macrophages, mast cells, and dendritic cells, as well as the epithelial cells that line the respiratory and intestinal tracts. TLRs are transmembrane glycoprotein receptors that trigger the synthesis and secretion of cytokines and activation of innate or adaptive immune responses. Mammals possess 10 or 12 different functional TLRs (Akira, 2003). The cell surface TLRs (TLR1, 2, 4, 5, 6 and 11) mainly recognize bacterial and fungal proteins, lipoprotein and lipopolysaccharides whereas the intracellular TLRs 3, 7, 8, 9 and 10 recognize viral and bacterial nucleic acids.

When a PAMP binds to its corresponding TLR, signals are passed to the cell. Multiprotein signaling complexes are formed, signal transduction cascades are initiated, and as a result, proinflammatory molecules are produced by the cell. The cell surface TLRs use different pathways than the intracellular TLRs. All TLRs except TLR3 use an adaptor protein called MyD88 to activate three major transcription factors, nuclear factor kappa-B (NF-kB), MAP kinase (MAPK), and IRF3.

NF- $\kappa B$ and MAPK activate the genes for three major proteins, interleukin-1 (IL-1), interleukin-6 (IL-6), and tumor necrosis factor-alpha (TNF- $\alpha$ ) IRF3 activates the gene for another protein, interferon- $\beta$. The proteins produced by these sentinel cells are all classed as cytokines. The cytokines are produced as inactive precursors and then activated by an enzyme called caspase- 1 . The production of caspase-1 is triggered by a protein complex called an inflammasome. Caspase- 1 is most important because it acts on the inactive precursors to generate active cytokines. These 
cytokines trigger the inflammatory response. TLRs that recognize bacterial molecules tend to trigger the production of cytokines optimized to combat bacteria; those that recognize viral molecules produce antiviral cytokines, and so forth. TLRs not only trigger innate responses such as inflammation but also begin the process of "turning on" the adaptive immune system. For example, stimulation of TLR4 makes macrophages and the dendritic cells, produce cytokines that are potent stimulators of immune cells. TLRs are also expressed on the bone marrow stem cells that are the source of leukocytes. Bacterial lipopolysaccharides binding to TLR4 on stem cells stimulates the bone marrow to increase leukocyte production. An increase in leukocyte numbers in the blood (the white cell count), is therefore a consistent feature of infectious diseases (Table 1).

\section{C-type lectin receptors (CLR)}

In innate immune system various cells are involved from dendritic cells to platelets expressed myriad CLRs which have pattern recognition ability (Geijtenbeek and Gringhuis, 2009). Lectins are proteins that bind carbohydrates. The C-type lectins require
$\mathrm{Ca}^{2+}$ for this binding, hence their name. CLRs can recognize the carbohydrates on bacteria, fungi, and some viruses. The major CLRs are dectin-1, dectin-2, and DEC205. These dectins recognize $\beta$-glucans on fungal cell walls and play an important role in antifungal defense.

They promote the intracellular destruction of fungi. Dectin-1 is expressed by bovine macrophages, monocytes, and dendritic cells. Bovine dectin- 2 is expressed in large amounts on Langerhans cells in the skin. DEC205 is expressed on bovine dendritic cells. Another C-type lectin, the macrophage mannose receptor, is expressed on macrophages and recognizes numerous diverse pathogens, such as the yeasts Candida albicans and Pneumocystis jiroveci, the protozoa Leishmania sp. and viruses such as bovine viral diarrhea virus (Table 2).

\section{Cytoplasmic PRRs}

\section{NOD-like receptors (NLR)}

Nucleotide-binding oligomerization domain (NOD)-like receptors (NLRs) is a family of PRRs that can also detect pathogens within the cytosol.

Fig.1 Location of intracellar and extracellular PRRs

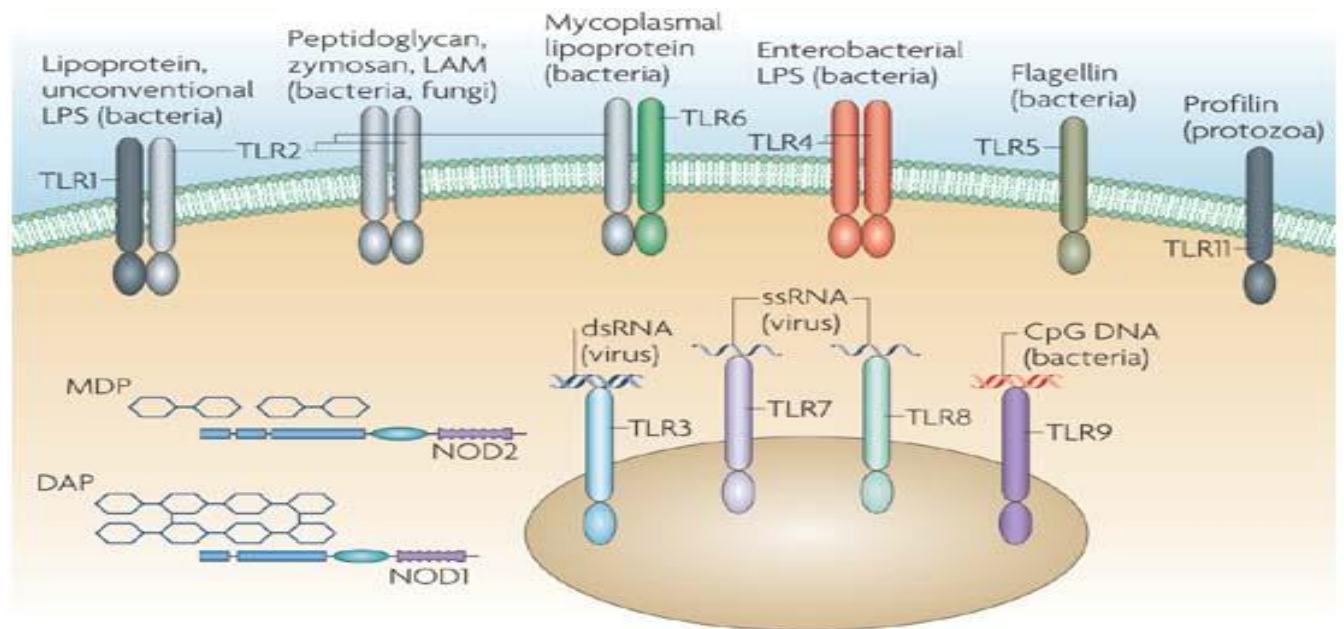


Table.1 Location of TLRs and their ligands

\begin{tabular}{|l|l|l|l|}
\hline TLR & Location & Ligand & Source of Ligand \\
\hline TLR1 & Cell surface & Triacylated lipoproteins & Bacteria \\
\hline TLR2 & Cell surface & lipoproteins & Bacteria, Virus, Parasites \\
\hline TLR3 & Intracellullar & dsRNA & Virus \\
\hline TLR4 & Cell surface & LPS & Bacteria, Virus \\
\hline TLR5 & Cell surface & Flagellin & Bacteria \\
\hline TLR6 & Cell surface & Diacylated lipoproteins & Bacteria, Virus \\
\hline TLR7 & Intracellullar & ssRNA & Virus, bacteria \\
\hline TLR8 & Intracellullar & ssRNA & Virus, bacteria \\
\hline TLR9 & Intracellullar & CpG DNA, dsDNA & Virus, bacteria, protozoa \\
\hline TLR10 & Intracellullar & Unknown & unknown \\
\hline TLR11 & Cell surface & $\begin{array}{l}\text { Toxoplasma profiling } \\
\text { like molecule }\end{array}$ & Protozoa \\
\hline TLR12 and TLR13 & Found in mice not in humans & & unknown \\
\hline
\end{tabular}

(Tizard, 2013)

Table.2 Location of RLRs, NLRs and CLRs with their ligands

\begin{tabular}{|c|c|c|c|c|}
\hline Receptor & Types & Location & Ligand & Source of Ligand \\
\hline RLRs & RIG-1 & Intracellular & Short dsRNA & RNA virus \\
\hline \multirow[b]{2}{*}{ NLRs } & NOD1 & Cytoplasm & Peptidoglycans & Bacteria \\
\hline & NOD2 & Cytoplasm & Muramyl dipeptide & Bacteria \\
\hline CLRs & Dectins & Cell surface & Glucans & Fungi \\
\hline Others & Mannose- fucose receptor & Cell surface & Glycoproteins & Bacteria \\
\hline
\end{tabular}

(Tizard, 2013)

Although TLRs and NLRs differ in their location and function, they share similar structures for microbial sensing and cooperate to trigger host responses to invaders. NOD1 recognizes bacterial peptidoglycans, whereas NOD2 recognizes muramyl dipeptide and serves as a general sensor of intracellular bacteria. Binding to either NLR activates the $\mathrm{NF}-\mathrm{\kappa B}$ pathway and triggers the production of proinflammatory cytokines. NOD2 also triggers the production of the antimicrobial proteins known as defensins.

The NLRs are proteins that initiate and regulate inflammatory and apoptotic responses. NLRs basic function is to detect the presence of endogenous or microbial molecules or stress responses and activate inflammatory caspases (e.g. caspase 1) which cause cleavage and activation of important inflammatory cytokines such as IL-1 and/or activate the NF- $\kappa \mathrm{B}$ signaling pathway to induce production of inflammatory molecules.

When pathogen binds to NLR they activate several cellular proteins which bind together to form large multiprotein complexes called inflammasomes. The inflammasome then recruits and activates Caspase- 1 which in turn acts on pro-IL-1, pro-IL-6, and TNF- $\alpha$ to generate the active forms. Several different inflammasomes have been characterized, generated by a different set of pathogens, containing slightly different subcomponents 
and thus presumably generating different cytokines and proinflammatory molecules (Davis et al., 2014).

\section{RIG-I-like receptors (RLRs)}

Retinoic acid inducible gene (RIG)-like receptors (RLRs) are another family of PRRs expressed within the cytosol. They recognize viral dsRNA. Because viral dsRNA differs structurally from mammalian RNA, RLRs can discriminate between viral and normal mammalian RNA. Once triggered, the RLRs activate caspases and trigger signaling pathways, leading to the production of type I IFNs. RIG-I-like receptor intracellular recognition (Ireton and Gale, 2015) of viral double-stranded (ds) and single stranded RNA mediated by a group of RNA helicases which in turn recruit factors to activate antiviral gene programs. Three such helicases have been described in mammals: RIG-I and MDA5 (recognizing 5'triphosphate-RNA and dsRNA, respectively) which activate antiviral signaling and LGP2, which appears to act as a dominantnegative inhibitor.

\section{Secreted PRRs}

There is a distinct class of PRRs which do not remain associated with the cell. Complement receptors, collectins, ficolins, pentraxins such as serum amyloid and C-reactive protein, lipid transferases and peptidoglycan recognition proteins (PGRs) are all secreted as proteins (Wang et al., 1998). A major PRR of the innate immune system, mannan-binding lectin (MBL) - collectin is capable of detecting wide range of bacteria, viruses, fungi and protozoa.

MBL detects and binds certain sugar groups on the surface of microorganisms but also binds phospholipids, nucleic acids and nonglycosylated proteins (Dommett et al., 2006). Once bound to the ligands the MBL recruit MASP1 and MASP2 and initiate the lectin pathway of complement activation and destroy the invader.

\section{Soluble pattern-recognition receptors}

Although the TLRs, NLRs, and RLRs are expressed on cell surfaces, many soluble PRRs can also bind PAMPs. Three extracellular lectin families, P, S, and C-type lectins, are involved in innate immunity.

P-type lectins are also called pentraxins. Two are important acute-phase proteins: C-reactive protein (CRP) and serum amyloid $\mathrm{P}$ (SAP). They are called acute phase proteins because their blood levels rise greatly during infections or after trauma. Pentraxins have multiple biological functions, including activation of complement and stimulation of leukocytes. They bind to microbial carbohydrates such as LPS in a calcium-dependent manner and activate the classical complement pathway by interacting with $\mathrm{C} 1 \mathrm{q}$.

S-type lectins are extracellular galectins. Their name derives from their specificity for galactosides. They play a role in inflammation by binding leukocytes to the extracellular matrix.

C-type lectins (collectins), are a very large family of proteins with many different roles. All require calcium for binding to carbohydrates. Each end of a collectin molecule has a distinct function; the C-terminal domain binds to carbohydrates, whereas the $\mathrm{N}$-terminal domain interacts with cells or complement components, thereby exerting their biological effect. The most important of the soluble C-type lectins is mannose-binding lectin (MBL). MBL is found in high levels in serum. MBL has multiple carbohydrate-binding sites that bind oligosaccharides, such as $N$-acetyl glucosamine, mannose, glucose, galactose, and $N$ acetylgalactosamine. As a result, MBL binds very strongly to bacteria such as Salmonella enterica and Listeria monocytogenes. It binds to Escherichia coli with moderate affinity. MBL binds strongly to yeasts such as Candida albicans and Cryptococcus neoformans. It can bind viruses such as influenza $A$ as well parasites such as Leishmania sp. MBL plays an 
important role in activating the complement system. The collectins are especially important in the defense of young animals whose adaptive immune system is not capable of mounting an efficient response.

\section{References}

Akira, S. 2003. Mammalian toll-like receptors. Current Opinion in Immunology, 15:511.

Akira, S., Uematsu, S. and Takeuchi, O. 2007. Pathogen recognition and innate immunity. Cell, 124:783-801.

Bianchi, M. E. 2012. DAMPS, PAMPs and alarmins: all we need to know about danger. Journal of Leukocyte Biology, 81:1-5.

Blasius, A.L. and Beutler, B. 2010. Intracellular toll-like receptors. Immunity, 32:305315.

Davis, B. K., Wen, H. and Ting, J. P. Y. 2014. The Inflammasome NLRs in immunity, inflammation, and associated diseases. Annual Review of Immunology, 29:707-735.

Dommett, R. M., Klein, N. and Turner, M. W. 2006. Mannose-binding lectin in innate immunity: past, present and future. Tissue Antigens, 68:193-209.

Gardy, J.L., Lynn, D.J., Brinkman, F.S. and Hancock, R.E. 2009. Enabling a systems biology approach to immunology: focus on innate immunity. Trends in Immunology, 30:249-262.

Geijtenbeek, T. B. and Gringhuis, S. I. 2009. Signalling through C-type lectin receptors: shaping immune responses. Nature Reviews Immunology, 9: 465-479.

Ireton, R. C. and Gale, M. 2015. RIG-I like receptors in antiviral immunity and therapeutic applications. Viruses, 3:906919.

Janeway, C.A. 1989. Approaching the asymptote? Evolution and revolution in immunology. Cold Spring Harbor Symposia Quantum Biology, 54:1-13.

Kingeter, L. M. and Lin, X. 2012. C-type lectin receptor-induced $\mathrm{NF}-\kappa \mathrm{B}$ activation in innate immune and inflammatory responses. Cellular and Molecular Immunology-Nature, 9:105.

Lemaitre, B., Nicolas, E., Michaut, L., Reichhart, J. M. and Hoffmann, J. A. 1996. The dorsoventral regulatory gene cassette spätzle/toll/cactus controls the potent antifungal response in Drosophila adults. Cell, 86:973-983.

Medzhitov, R. 2007. Recognition of microorganisms and activation of the immune response. Nature, 449:819-826.

Medzhitov, R. 2008. Origin and physiological roles of inflammation. Nature, 454:428435.

Mogensen, T. H. 2009. Pathogen Recognition and Inflammatory Signaling in Innate Immune Defenses. Clinical Microbiology Reviews, 22:240.

Schroder, K. and Tschopp, J. 2010. The inflammasomes. Cell, 140:821.

Takeuchi, O. and Akira, S. 2010. Pattern recognition receptors and inflammation. Cell, 140: 805.

Wang, G.L., Ruan, D. L., Song, W. Y., Sideris, S., Chen, L., Pi, L.Y., Zhang, S., Zhang, Z., Fauquet, C., Gaut, B. S., Whalen, M.C. and Ronald, P.C. 1998. Xa21D encodes a receptor-like molecule with a leucine-rich repeat domain that determines race-specific recognition and is subject to adaptive evolution. The Plant Cell, 10:765-79.

\section{How to cite this article:}

Joycee Jogi, Varsha Sharma, Anju Nayak, Vandana Gupta, Poonam Shakya and Smita Bordoloi. 2018. Pattern Recognition Receptors (PRRs). Int.J.Curr.Microbiol.App.Sci. 7(09): 3400-3406. doi: https://doi.org/10.20546/ijcmas.2018.709.422 